morphia may be tried with good effect. But in this stage we must be on our guard against secondary inflammation; for the diminished irritation in which every organ has been, is a state liable to excessive irritation, on the recovery of the part originally affected, and which, if you produce it, will assuredly end in that degree of diminished irritation constituting inflammation. Therefore, now, the less we can do, the better. Stimulants should be withdrawn,-repose should be encouraged,-nourishment of the highest kind should be administered, and thus nature would have a fair chance of avoiding the breakers which were a-head of ler, when escaping the rock on which she had been so nearly wrecked.

There are one or two other points of a secondary nature which it may be as well to notice. The position of the patient is one such: of course the horizontal position must be enjoined; but that is not all, let him lie anyhow, rather than on his backeither side, or prone, if he please. He should also be assisted in getting out of bed, in turning, and not allowed to sit upright. The heart is too weak to allow of its being benefitted by the return of the blood being hindered by the sitting posture, and the brain likewise cannot spare any of the power which the blood in its ressels receives from the enfeebled heart. The temperature of the appartment must be kept steadily high.

The treatment which I have thus sketched will be looked at as embodying a general rule. Cases will occur where it would be losing time (to say the least of it,) in attempting to bleed. Every medical man has patients which he knows cannot bear the loss of blood, and such he would not hesitate to consider unfit subjects for that renedy; and so likewise will every other suggestion be subjected to the judgment of the medical attendant, and adapted to the individual's case before him. It must also be remarked, that with such a disease as Asiatic cholera attacking certain individuals, the result must necessarily be fatal; and well will it be for the practitioner when his knowledge of his patient's constitution enables hin to judge correctly of what can be borne by him. It seems hardly necessary to say anything of the treatment of any secondary affection which may arise to interrupt and prolong the recovery of the patient.

\section{OBSERVATIONS ON SCURVY}

\author{
AS IT WAS DEVELOPED
}

IN BATH AND ITS NEIGHBOURHOUD, IN THE SPRING OF 1847.*

By John Barrett, Esq., F.R.C.S.

(Read at the Quarterly Meeting of the Bath and Bristol Branch of the Provincial Medical and Surgical Association, December 21, 1848.)

Of few diseases can it be more truly said that prevention is better than cure, than of scurvy. Under favourable circumstances its cure is tedious, and the means of cure are not always to be met with in the circumstances under which it occurs. But it is not

- Continued from page 177. only that its cure is tedious, and sometimes for a time impossible, it is at:ended with consequences of a very dangerous character,-e.g., the effect it produces on old surgical cases; the re-opening of wounds; the disunion of fractures; and it certainly lays the foundation of fatal chest and other diseases, by breaking down the constitution. If we judge of it by the number of deaths in its recognized appearance, we may probably consider it a matter of secondary importance, but this, I have before said, is a very inadequate manner of judging. It is not a disease of sudden occurrence, it requires months' duration of the preparatory circumstances. But as there can be no doubt that it has appeared, and frequently, (where even the profession may not have suspected, it is constantly occurring,) is there, I would ask, no reason for fearing that a disease which has decimated armies, and destroyed fleets, may in our day regain a fearful and destructive influence, if we do not remove the causes of its occurrence, or if by foolish directions we increase them. There is much wisdom in that part of the address from the College of Physicans on cholera, - "The Committee do not recommend that the public should abstain from the moderate use of well cooked green vegetables, and of ripe or preserved fruits. A certain proportion of these articles of diet is, with most persons, necessary for the maintenance of bealth, and there is reason to fear that if they be generally abstained from, now that the potato crop has in a great measure failed, many persons, especially among. the poor in large towns, will fall into that ill condition, which, in its highest degree, is known as scurvy, and that they will in consequence be the readier victims of cholera."

There is one point to which, I am ashamed to say, I have not directed either my own, or my correspondent's, attention. The Registrar-General, in his return for the quarter ending December 31st, 1846, says,_- "No mention is made of the potato disease having had any direct connection with the mortality. The potato in a state of partial disease, has no doubt, been extensively consumed, without giving rise to any specific malady in man, or indeed having any appreciable connection with the disorders of the bowels, and fever, which were prevalent about the time the last crop came into use." I asked one gentleman, who said some of his scurvy cases had not been debarred potatoes, whether they had been good or diseased, but this he could not inform me. A poor woman, aged 76, applied to me, October 31st, this year, for griping pains in the bowels, increased on pressure; evacuations once a day, relaxed; white dry tongue, and weak pulse. Three weeks before she ate some potatoes, which were very watery and yellow, and had not been well since. She took small doses of calomel and opium for a few days, which cured her.

It is a question of much interest-what is the nature 
of those changes in the animal economy which give rise to the symptoms of scurvy. Its most efficient external cause, the absence of certain organic acids from the food is so definite and determined, that if there be any cases in which the discoveries of the chemist can be brought to bear on the investigations of the pathologist we should expect this would be one of them. Yet, hitherto, there seems to have been hardly an attempt at a pathological rationale of scurvy. The idea that the globules of the blood are in it, dissolved in the serum, is abundantly disproved by the fact that, scurvy blood will separate as rapidly into clot and serum as healthy blood; but $I$ am not aware of any attempt to explain how it is that the absence of an organic acid from the food is attended with an improper excess of the water, fibrin, albumen, and the salts, with a deficiency of the hæmatosine of the blood, for this seems to constitute the morbid alterations of the blood in scurrs. Dr. Budd remarks, "The study of scurvy is most instructive to the pathologist, showing as it does, the variety and importance of the effects which may result from a primary alteration in the quality of the blood, independently of any morbid change in the solids." I have no wish to underrate the importance of these morbid alterations in the blood, but I question whether future investigations on this subject may not show that this conclusion is rather too exclusive.

There is another fluid in the body possessing properties strongly distinguishing it from the blood, for a knowledge of which we are indebted to Liebig, and thongh attention has only lately been directed to it, there is reason for believing that it plays a very important part in the animal economy. "Besides the blood and fluids contained in the lymphatic and absorbent ressels, there exists in flesh a considerable quantity of a peculiar fluid, enclosed either in peculiar minute ressels, or more probably in the cells of the fibrous and cellular tissues. To this liquid Liebig gives the name of the juice of flesh, and he has lately subjected it to a minute investigation with very important results."-("Turner's Chemistry, 1848, p. 1280.) The important difference between this ftuid and the blood is, that whereas, the blood is alkaline, cortaining phosphate of sods-an alkaline salt; the juice of flesh is acid, containing phosphate of potash, and acid salt, and several organic acids, the most important of which is the lactic. Now, it is interesting to remember that though some animals will live on food wanting in the regetable organic acids, man will not. The carniverous animals will of course lire on flesh only which contains lactic acid, but there is abundant proof that man will not long subsist on flesh or even milk without scurvy, but must have a supply of food containing regetable organic acids. We know that these organic acids, composed as they are, of the same nltimate principles, -i. e., carbon, hydrogen, and oxygen in different proportions, are convertible into one another;-thus, citric acid, "when melted with an excess of caustic potash, is resolved into oxalic and acetic acids and water, which is merely a different arrangement of the same elements."-“(Turner's Chemistry, p. 1039.") It is surely, therefore, no rery bold hypothesis that in such animals as the carniverous, the lactic acid may be derived from their food as lactic acid, but that the organization in man may require that it be derived from other organic acids which undergo chemical change after they have entered the body; and that unless such a change go on health cannot be kept up, but certain morbid consequences follow. And if the gastric juice be " very nearly identical with the juice of flesh, or fluid of the muscles," ("Turner's Chemistry," p. 1293,) we can easily understand how a deficiency of the means of supplying the animal system with lactic acid might lead to a disease presenting features of depraved nutrition like scurvy. Again, Liebig bas shown that only a small fraction of the whole amount of bile can be detected in any shape in the faces, and that the bile is unquestionably reabsorbed in the intestinal canal and re-enters the circulation, where it $800 \mathrm{n}$ dis appears; and if it be correct, as is supposed; "that the bile is resolved into lactic acid and other products before it is finally consumed," ("Turner's Chemistry," p. 1314.") any derangement of the digestive function, any affection of the liver, such as scurvy has been considered to present, would speedily react on the system and diminish the quantity of lactic acid, and consequently the chemical actions involved in its presence, and bence might follow the necessity of relieving this viscus which has been so strongly insisted on. Again, in his "Animal Chemistry," Liebig endeavoured to show, taking the then admitted formula for bile and for blood, " that the latter fluid, with the addition of a little water and oxygen, might be resolved into choleric acid and urate of ammonia; - that is, into bile and urine." Now, I would remark, that lactic acid is acetic acid, with two proportionals of water and one of oxygen. If, then, we were to suppose that in the liver this quantity of oxygen and water is removed from the lactic acid then existing in the juice of flesh and acting on the blood, we should get as products, bile, acetic acid and urine; the bile discharged in the alimentary canal, the acetic acid carried to the gastric juice, the urate of ammonia, by further oxidization, yielding finally carbonic acid, ammonia, and urea. But if there should be a deficiency in the quantity of lactic acid, of course this chemical change will be interfered with, the blood would not be exhausted in the liver, and might not this lead to the congestion of that organ?

In scurvy blood, whilst fibrin and albumen are in excess, hrmatosine is deficient in quantity. But albumen and fibrin may be considered elementary 
parts of the body, whilst hæmatosine, though possessing many properties in common with albumen, is probably the result of chemical change of one or both, and if this be so, its deficiency and their excess would seem to imply a deficiency of chemical action.

Another morbid condition in the blood of scurry is the excess of the salts. Now, the nuost important salt of the blood is the phosphate of soda, which gives it its alkaline character, whilst the juice of flesh contains the phosphate of potash, an acid salt which is "the only phosphate that can be formed by an animal from food containing salts of potash without soda, such as inland plants." There can be no doubt that the function of the acid salt, the phosphate of potash in the juice of flesh, and apparently also in the gastric juice, is as important as that of phospate of soda in the blood," and if the want of regetable food should deprive the animal of the proper means of forming this, the balance of acidity and alkalinity of juice of flesh, and blood, would be interfered with, and all chemical changes involred in such proper balance and proportion might be also. The gastric juice contains free phosphoric acid, but if the food be principally salt meat, and wanting in fresh vegetables, it will necessary expose soda, not potash, to the acid, and may thus lead to that combination which gives the alkuline character to the blood, and may lead to the excess of the salts found in scurvy blood, whilst it may deprire the juice of flesh of its necessary acidity, and break up the proper balance as before observed. It is a remark worth consideration, the salting of meat is detrimental to its nutritive qualities, becanse an exchange takes place between the salt and the juice," that is, not only is tos much salt carried into the stomach, but it is also deprived of the acids contained in the juice of fesh.

We bave then, I think, reason for believing that in scurry there must be very considerable suspension of those chemical changes which go on in health, but chemical action never takes place without a development of electricity. Our attention was called to this subject by Dr. Daries, at our summer district meeting, in a paper on "Traumatic Paralysis," treated by electro-magnetism. He then referred to the interesting facts relative to the development of electricity in the animal economy, bronght forward by Liebig and Matteucci, and of which the following mention is found in "Turner's Chemistry," p. 1320 :- "Another probable function of the substances which give acidity to the juice of flesh and alkalinity to the blood, is the production of electrical currents. It has been shown by Matteucci, that such currents exist in the body, and we can easily see how they may arise when we observe two fluids, one acid, and the other alkaline, separated by a membrane permeable to one or both, and the finids in contact with muscle, and with nerrous matter. At the request of Liebig, Buff constructed piles of discs of pasteboard, steeped in blood, with slices of muscle and brain, which showed a powerful current from the blood to the muscle." But supposing the alkaline qualities of the blood to be increased (as by analysis we know they are in scurvy,) and the acid qualities of the juice of flesh to be decreased, should we not expect that there would be a coustant decrease of electrical development. Now, from Dr. Davies's paper, for to this I must refer, not having read Matteucci's lectures, it would appear, that in rheumatism there is a marked deficiency in electricity; that in all muscles there is a constant current of free electricity, bearing a direct ratio to the vigour of the animal, and the degree of development of the muscular structure. But what symptoms are more constant or more strongly marked in scurvy than the absence of muscular vigour, and the presence of muscular pain, debility, and rheumatism. And may we not conclude, that in the suspension of certain chemical actions in the animal economy, the derangement of certain states of chemical proportion, in the different fluids, and in the consequent interruption of electrical development, to be found at once a rationale of the morbid changes which take place in the structures of the body, and of the symptoms of the disease. Dr. Davies also states that the higher the animal is in the scale of being, the greater is the amount of electricity developed. I have called your attention to an important difference between the carnirorous animals and man, who stands highest in the scale of organization; they will continue in a state of health living on flesh containing an organic acid-the lactic acid; but man requires a vegetable organic acid, and one which, if it sbould be a source of lactic acid contained in his muscular fluid, must be so by undergoing chemical change, and thereby giving rise to electrical development. And may we not, therefore, conclude that the necessity for this superior degree of electrical development, connected, as it is, with his superior organization, is one reason why he requires in his food a vegetable organic acid ?

'These remarks have extended to such a length that I fear I shall be looked on as hopelessly affected with the cacoethes scribendi. In palliation, I must remind you, that $I$ have been bringing before you the opinions of a number of medical men, on a question of really national interest. Some credit, however, I must take to myself for sparing you the infliction of all $\mathrm{my}$ scribblings. I may say with Pope,- "For what I have published, I can only hope to be pardoned; but for what I have burned, I deserve to be praised. On this acconnt the world is under some obligation to me." 